\title{
A systematic review and meta-analysis of adherence to physical activity interventions among three chronic conditions: cancer, cardiovascular disease, and diabetes
}

Tiffany Bullard ${ }^{1}$, Mengmeng Ji', Ruopeng An¹, Linda Trinh², Michael Mackenzie ${ }^{3}$ and Sean P. Mullen ${ }^{1 *}$ (D)

\begin{abstract}
Background: Physical activity is effective for the prevention and treatment of chronic disease, yet insufficient evidence is available to make comparisons regarding adherence to aerobic physical activity interventions among chronic disease populations, or across different settings.

The purpose of this review is to investigate and provide a quantitative summary of adherence rates to the aerobic physical activity guidelines among people with chronic conditions, as physical activity is an effective form of treatment and prevention of chronic disease.

Methods: Randomized controlled (RCTs) trials where aerobic physical activity was the primary intervention were selected from PsychInfo, PubMed, CINAHL (Cumulative Index to Nursing and Allied Health Literature), Clinical Key, and SCOPUS from 2000 to 2018. Studies were included if the program prescription aligned with the 2008 aerobic physical activity guidelines, were at least 12 weeks in length, and included adult participants living with one of three chronic diseases. The data was extracted by hand and the PRISMA (preferred reporting items for systematic review and meta-analysis) guidelines were used to evaluate risk-of-bias and quality of evidence. Data were pooled using random-effect models. The primary outcome measure was program adherence and the secondary outcome measures were dropout and setting (e.g. home vs. clinic-based). Pooled effect sizes and 95\% Cils (confidence intervals) were calculated using random-effect models.
\end{abstract}

Results: The literature search identified 1616 potentially eligible studies, of which 30 studies (published between 2000 and 2018, including 3,721 participants) met the inclusion criteria. Three clinical populations were targeted: cancer $(n=14)$, cardiovascular disease $(n=7)$, and diabetes $(n=9)$. Although not statistically significant, adherence rates varied across samples $(65,90$, and $80 \%$, respectively) whereas dropout rates were relatively low and consistent across samples $(5,4$, and $3 \%)$. The average adherence rate, regardless of condition, is $77 \%(95 \% \mathrm{Cl}=0.68,0.84)$ of their prescribed physical activity treatment. The pooled adherence rates for clinic-based and home-based programs did not differ $(74 \%[95 \% \mathrm{Cl}, 0.65,0.82]$ and $80 \%$ [95\% Cl, 0.65, 0.91], respectively).

Conclusions: The current evidence suggests that people with chronic conditions are capable of sustaining aerobic physical activity for $3+$ months, as a form of treatment. Moreover, home-based programs may be just as feasible as supervised, clinic-based physical activity programs.

Keywords: Exercise adherence, Dropout, Chronic disease, Cancer, Cardiovascular disease, Diabetes

\footnotetext{
* Correspondence: spmullen@illinois.edu

'University of Illinois at Urbana-Champaign, Champaign, USA

Full list of author information is available at the end of the article
}

(c) The Author(s). 2019 Open Access This article is distributed under the terms of the Creative Commons Attribution 4.0 International License (http://creativecommons.org/licenses/by/4.0/), which permits unrestricted use, distribution, and reproduction in any medium, provided you give appropriate credit to the original author(s) and the source, provide a link to the Creative Commons license, and indicate if changes were made. The Creative Commons Public Domain Dedication waiver (http://creativecommons.org/publicdomain/zero/1.0/) applies to the data made available in this article, unless otherwise stated. 


\section{Background}

Chronic disease is the leading cause of death in America and almost $50 \%$ of adults have one or more chronic health conditions [1]. Increasing physical activity has been shown to be an effective form of treatment and prevention of chronic disease [2-4]. The benefits of regular physical activity include, but are not limited to, weight control, strengthening of muscles and bones, increases in balance and general physical functioning, and improvements in mental health [5] and health-related quality of life [6]-all factors negatively affected by chronic disease. Current public health guidelines [7] recommend $150 \mathrm{~min}$ of moderate-to-vigorous aerobic exercise per week. However, it has previously been reported that only $35 \%$ of women after breast cancer diagnosis [8], 32\% of those with cardiovascular disease (CVD) [9], and 46\% of people with diabetes met physical activity guidelines [10]. These low adherence rates are not altogether surprising, as individuals with chronic disease have many barriers (i.e. fatigue, pain) to continued physical activity participation relative to those without chronic disease.

Typical treatment for chronic disease involves managing symptoms with medication and accounts for $86 \%$ of the total health care expenses in the United States [11]. There is evidence within the medical community that physical activity is comparably effective as an additional treatment of disease [12] and lowering the risk of mortality [13] relative to standard treatment methods (e.g. medications, surgery, chemotherapy and radiation). However, research trials can vary substantially in their methodologies as well as their setting (clinic- vs. home-based). Both settings have unique advantages. Clinic-based programs often provide more detailed and intensive supervision, whereas home-based programs typically provide more autonomy (e.g., more choices regarding training schedule, fewer transportation-related barriers to receive intervention). In a review of physical activity interventions designed for older adults, Conn et al. [14] found a greater effect for clinic-based interventions $(d=.26)$ relative to home-based programs. It is possible that the supervision provided in the clinic-based studies resulted in greater adherence to the program. It cannot be assumed that patients will uniformly adhere to any structured physical activity program, irrespective of their condition. Specifically, do patients' adherence levels vary across chronic conditions (e.g., cardiovascular vs. metabolic) or type of program (e.g., clinic or home-based)? Answering these questions is essential for practitioners and researchers, as both are interested in understanding how to optimize the delivery of physical activity as medicine as an adjuvant treatment for disease.
The three most commonly studied chronic diseases in the context of physical activity interventions are cancer, CVD, and diabetes. Physical activity has been shown to be an effective treatment for each of these diseases and current evidence suggests exercise has a positive effect on patient quality of life, physical functioning, and fatigue compared to usual care. For example, Gerritsen and Vincent [15] examined the evidence from randomized controlled trials involving cancer patients in a systematic review and meta-analysis and determined that exercise significantly improved self-esteem, physical performance and functioning, fatigue, and social functioning. According to an observational study of 2987 women diagnosed with breast cancer [16], those who participated in regular physical activity (9+ MET (metabolic equivalent task)-hours per week) saw reductions in breast cancer mortality (relative risk: 0.50, 95\% CI: 0.340.74). Similarly, Anderson et al. [17] reviewed exercise-based cardiac rehabilitation programs and, relative to usual care, exercise improved quality of life, reduced hospital admissions post-treatment (relative risk: 0.82, 95\% CI: 0.70 to 0.96 ), and reduced cardiovascular mortality (relative risk: $0.74,95 \% \mathrm{CI}: 0.64$ to 0.86 ), independent of study quality, setting, and publication date. Likewise, Umpierre et al. [18] provided substantial evidence that structured exercise training is associated with reduced levels of hemoglobin $\mathrm{A}_{1 \mathrm{C}}\left(\mathrm{HbA}_{1 \mathrm{C}}\right)(-0.67 \%$; 95\% CI: $-0.84 \%$ to $-0.49 \%, p<0.001)$, as well as reduced risks for diabetes-related complications in patients with type 2 diabetes. Hu et al. conducted a longitudinal study of 3708 patients with type 2 diabetes [19] and showed a reduction in mortality risk across low, moderate, and high physical activity levels (relative risk: 1.00, 0.59, and 0.49 , respectively). Altogether, there is substantial evidence for using exercise as medicine [20] (i.e. treating disease, lowering mortality), but comparisons regarding adherence to aerobic exercise prescriptions across these conditions, and between clinic- and home-based settings, have not been made. Given that exercise can serve as a standalone and complementary medicine, more research is needed examining the relative acceptability of activity prescriptions across populations.

In this systematic review and meta-analysis, we aimed to test the potential differences in adherence and dropout rates among patients involved in aerobic physical activity interventions. We hypothesized that cancer patients would exhibit the lowest adherence rates with the knowledge that very few cancer patients meet the recommended physical activity guidelines (2008 or 2018) for aerobic exercise, and given the long-lasting and debilitating effects of chemotherapy and radiation treatment (e.g., fatigue, cognitive impairment) [21] compared to CVD or diabetes. In addition, we also hypothesized higher adherence associated with clinic-based programs 
relative to home-based programs because there is arguably more supervision and accountability in such programs.

\section{Methods}

\section{Study inclusion criteria}

RCTs with an aerobic (only) exercise intervention were included in the review. Specifically, trials must have included an explicit program prescription aligned with the 2008 Physical Activity Guidelines for aerobic exercise (i.e., a minimum of $150 \mathrm{~min}$ per week). Also, trials that included adult participants (age 18+), and published results met study inclusion criteria. Physical activity interventions lasting at least 12 weeks were included to align with expected dropout trends previously reported in the literature among people with and without clinical conditions $[22,23]$. Studies were included if rates of adherence and dropout were explicitly reported. Author confirmation was required if study data was not reported in sufficient detail. In this study, adherence was defined as meeting the aerobic physical activity recommendations of $150 \mathrm{~min} /$ week across the study duration (expressed as a percentage). Dropout was defined as participants who formally withdrew or left the study and did not return (e.g. non-responders who did not officially relinquish their consent to participate). This threshold was determined as one month or longer as a substantial period of time, whereas less than one month could reflect brief illness or vacation. If the paper provided definitions that varied from the ones above, or did not explicitly provide values, the author was contacted and asked to provide the information as requested in order to standardize the data. The intervention frequency, intensity, type, and duration were recorded for each study and each intervention was identified as either home- or clinic-based. Inclusionary criteria allowed for all types of cancer, $\mathrm{CVD}$, and diabetes, as long as patients were currently diagnosed with one of the targeted diseases. Although type-1 diabetes was not exclusionary, all diabetes studies in this review included populations with type-2 diabetes. The type of cancer patients' treatment (e.g. chemotherapy, radiation, surgery) was not exclusionary.

\section{Study exclusion criteria}

Studies were excluded for a variety of reasons. The most common reason for exclusion was that exercise was not the primary intervention. For example, a lifestyle intervention study [24] provided counseling with the primary outcome being weight loss. Although physical activity was assessed within the study, it was not a primary outcome of the intervention. Exclusionary criteria did not allow for healthy populations (studies that did not include patients with cancer, CVD, or diabetes), or if the patients did not currently have the disease (e.g., cancer survivors).

\section{Search strategy}

The research databases PsychInfo, PubMed, CINAHL, Clinical Key, and SCOPUS were searched limiting the publication range from 2000 to 2018. The keywords were 'physical activity' and 'exercise', with 'adherence,' 'compliance,' or 'drop out' for 'cancer,' 'cardiovascular disease,' 'coronary disease,' 'coronary risk,' or 'diabetes'. Filters were used to select only RCTs when available. Additional studies were added through a manual search targeting existing meta-analyses and systematic reviews of physical activity interventions for specific clinical populations. Experts within the field were contacted for any published papers the authors may have omitted.

\section{Study selection}

The PRISMA-P (preferred reporting items for systematic review and meta-analysis protocols) guidelines [25] were followed in the reporting of this systematic review protocol (see Figs. 1 and 2). The search was conducted from February 2016 to October 2018. Titles and abstracts containing the key words were searched more thoroughly to ensure the selection criteria was met. Articles needed to be written in English and included adult samples only to be considered for the review. The included and excluded publications were subsequently reviewed by a second author (S. P. M.) until $100 \%$ consensus was reached regarding the final sample of studies to be included in the analysis. Disagreements about papers meeting all requirements were discussed amongst the authors until a consensus was reached.

\section{Data extraction}

The data for this review and meta-analysis was extracted by hand and stored within a Microsoft Excel spreadsheet. Data were extracted by the first author and checked for alliance with search criteria by the senior author. Data included information about the publication (authors, year, title), participant characteristics (number, age, gender, disease type), intervention characteristics (home- vs. clinic-based, length), and measurement characteristics (adherence definition and rates, dropout definition and rates, confirmation of data by author, intention-to-treat analysis). Authors were contacted if a measure was not explicitly reported within the publication.

\section{Analysis and synthesis}

A meta-analysis was performed to estimate the pooled difference in dropout rates between the intervention and control group and the pooled adherence rate in the intervention group. In addition, separate pooled effect 


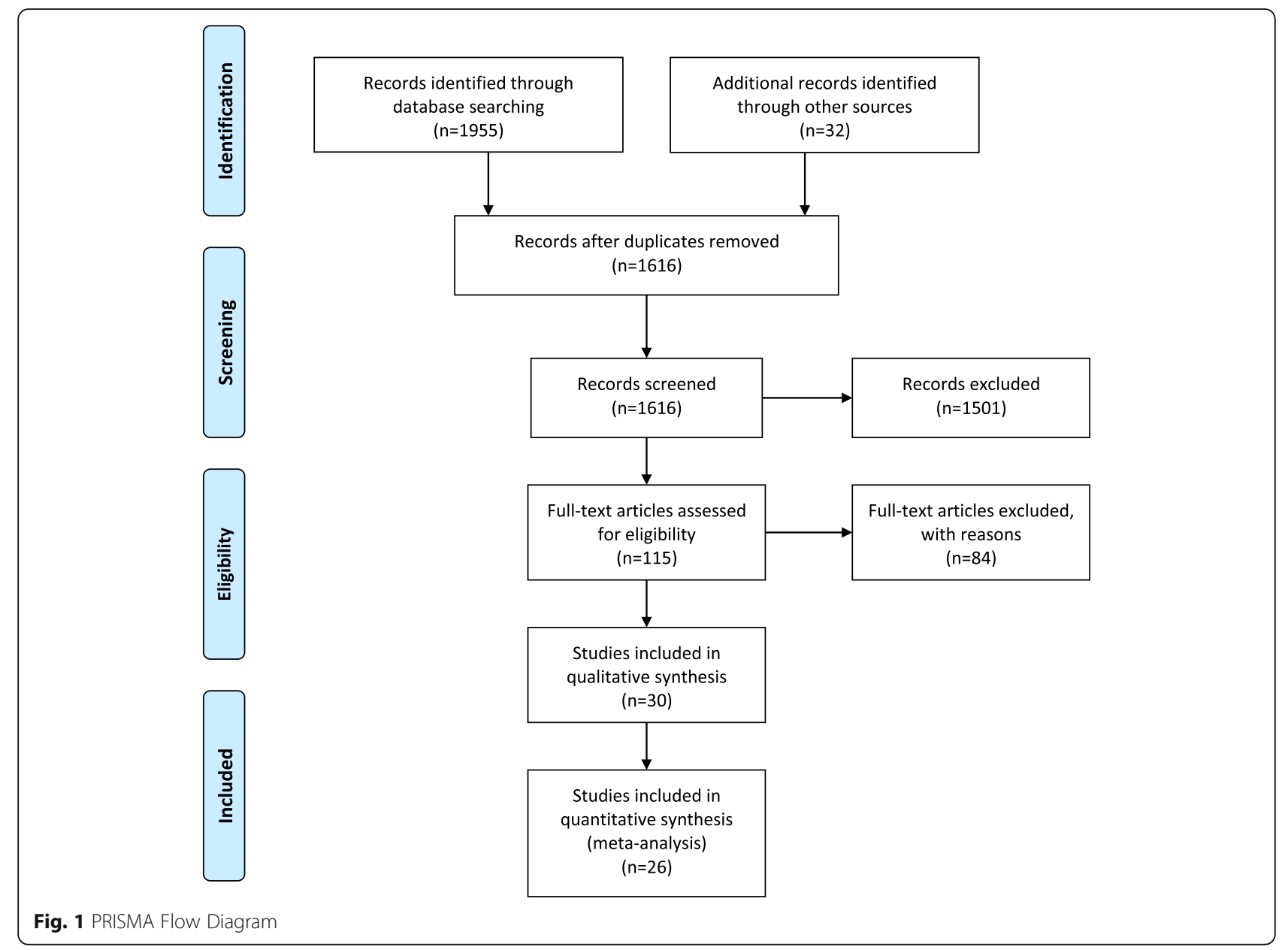

sizes were estimated based on studies stratified by disease type (i.e., cancer, CVD, and diabetes). A random-effect model was estimated given a $p$-value less than 0.05 from the Cochran's Q test or an $\mathrm{I}^{2}$ statistics greater or equal to $50 \%$; otherwise, a fixed-effect model was estimated. Meta-regressions were conducted to examine the potential heterogeneities in differential dropout rates between the intervention and control group and adherence rate in the intervention group attributable to different disease type. The independent variables in both meta-regressions were two categorical variables for CVD and type 2 diabetes, with cancer as their common reference group. Additional meta-regressions were conducted to assess dropout/adherence rates in relation to intervention duration (measured by a continuous variable for trial length in weeks), intention to treat (ITT) status (measured by a dichotomous variable for intervention conducted following the ITT principle), age (measured by a continuous variable for mean age of the study sample), and intervention setting (measured by a dichotomous variable for home-based intervention, with clinic-based intervention as the reference group). If studies with multiple intervention groups were included in the review, only the aerobic exercise group was included included in the analysis in comparison to the usual care group.

Publication bias was assessed using the Begg's test and Egger's test. All analyses used two-sided tests, and $p$-values less than 0.05 were considered statistically significant. All statistical analyses were conducted using Stata 15.1 SE version (StataCorp, College Station, TX).

\section{Results}

\section{Characteristics of included studies}

Following a comprehensive literature search, there were 1616 eligible studies, published between 2000 and 2018. The search and selection of articles are summarized in the study flow diagram (Fig. 1). Study characteristics can be found in Table 1 . The median sample size was 81 participants (range 14 to 606). A total of 3,721 participants were included in this review. The 30 studies included examined cancer $(n=14), \operatorname{CVD}(n=7)$, and diabetes $(n=9)$. All studies included patients that were currently diagnosed with any type of cancer, CVD, or diabetes. Among the cancer studies, there were four types included in this review: breast, prostate, colorectal, and ovarian. The CVD studies included heterogeneous 


\begin{tabular}{|c|c|c|c|}
\hline Section/topic & \# & Checklist item & $\begin{array}{l}\text { Reported } \\
\text { on page \# }\end{array}$ \\
\hline \multicolumn{4}{|l|}{ TITLE } \\
\hline Title & 1 & Identify the report as a systematic review, meta-analysis, or both. & $\begin{array}{l}\text { Manuscript } \\
\text { title }\end{array}$ \\
\hline \multicolumn{4}{|l|}{ ABSTRACT } \\
\hline Structured summary & 2 & $\begin{array}{l}\text { Provide a structured summary including, as applicable: background; objectives; data sources; study eligibility criteria, } \\
\text { participants, and interventions; study appraisal and synthesis methods; results; limitations; conclusions and } \\
\text { implications of key findings; systematic review registration number. }\end{array}$ & $\begin{array}{l}\text { Abstract } \\
\text { section }\end{array}$ \\
\hline \multicolumn{4}{|l|}{ INTRODUCTION } \\
\hline Rationale & 3 & Describe the rationale for the review in the context of what is already known. & 2 \\
\hline Objectives & 4 & $\begin{array}{l}\text { Provide an explicit statement of questions being addressed with reference to participants, interventions, comparisons, } \\
\text { outcomes, and study design (PICOS). }\end{array}$ & 3 \\
\hline \multicolumn{4}{|l|}{ METHODS } \\
\hline Protocol and registration & 5 & $\begin{array}{l}\text { Indicate if a review protocol exists, if and where it can be accessed (e.g., Web address), and, if available, provide } \\
\text { registration information including registration number. }\end{array}$ & 5 \\
\hline Eligibility criteria & 6 & $\begin{array}{l}\text { Specify study characteristics (e.g., PICOS, length of follow-up) and report characteristics (e.g., years considered, } \\
\text { language, publication status) used as criteria for eligibility, giving rationale. }\end{array}$ & 3-4 \\
\hline Information sources & 7 & $\begin{array}{l}\text { Describe all information sources (e.g., databases with dates of coverage, contact with study authors to identify } \\
\text { additional studies) in the search and date last searched. }\end{array}$ & 5 \\
\hline Search & 8 & $\begin{array}{l}\text { Present full electronic search strategy for at least one database, including any limits used, such that it could be } \\
\text { repeated. }\end{array}$ & 5 \\
\hline Study selection & 9 & $\begin{array}{l}\text { State the process for selecting studies (i.e., screening, eligibility, included in systematic review, and, if applicable, } \\
\text { included in the meta-analysis). }\end{array}$ & 5 \\
\hline Data collection process & 10 & $\begin{array}{l}\text { Describe method of data extraction from reports (e.g., piloted forms, independently, in duplicate) and any processes } \\
\text { for obtaining and confirming data from investigators. }\end{array}$ & 5 \\
\hline Data items & 11 & $\begin{array}{l}\text { List and define all variables for which data were sought (e.g., PICOS, funding sources) and any assumptions and } \\
\text { simplifications made. }\end{array}$ & 5 \\
\hline $\begin{array}{l}\text { Risk of bias in individual } \\
\text { studies }\end{array}$ & 12 & $\begin{array}{l}\text { Describe methods used for assessing risk of bias of individual studies (including specification of whether this was } \\
\text { done at the study or outcome level), and how this information is to be used in any data synthesis. }\end{array}$ & 6 \\
\hline Summary measures & 13 & State the principal summary measures (e.g., risk ratio, difference in means). & 6 \\
\hline Synthesis of results & 14 & $\begin{array}{l}\text { Describe the methods of handling data and combining results of studies, if done, including measures of consistency } \\
\text { (e.g., (l) for each meta-analysis. }\end{array}$ & 6 \\
\hline Risk of bias across studies & 15 & $\begin{array}{l}\text { Specify any assessment of risk of bias that may affect the cumulative evidence (e.g., publication bias, selective } \\
\text { reporting within studies). }\end{array}$ & 7 \\
\hline Additional analyses & 16 & $\begin{array}{l}\text { Describe methods of additional analyses (e.g., sensitivity or subgroup analyses, meta-regression), if done, indicating } \\
\text { which were pre-specified. }\end{array}$ & 6 \\
\hline \multicolumn{4}{|l|}{ RESULTS } \\
\hline Study selection & 17 & $\begin{array}{l}\text { Give numbers of studies screened, assessed for eligibility, and included in the review, with reasons for exclusions at } \\
\text { each stage, ideally with a flow diagram. }\end{array}$ & 7 \\
\hline Study characteristics & 18 & $\begin{array}{l}\text { For each study, present characteristics for which data were extracted (e.g., study size, PICOS, follow-up period) and } \\
\text { provide the citations. }\end{array}$ & 7 \\
\hline Risk of bias within studies & 19 & Present data on risk of bias of each study and, if available, any outcome level assessment (see item 12). & 8 \\
\hline Results of individual studies & 20 & $\begin{array}{l}\text { For all outcomes considered (benefits or harms), present, for each study: (a) simple summary data for each } \\
\text { intervention group (b) effect estimates and confidence intervals, ideally with a forest plot. }\end{array}$ & $\begin{array}{l}\text { Table 1; } \\
\text { Figures } 1 \\
\text { and } 2\end{array}$ \\
\hline Synthesis of results & 21 & Present results of each meta-analysis done, including confidence intervals and measures of consistency. & 7-8 \\
\hline Risk of bias across studies & 22 & Present results of any assessment of risk of bias across studies (see Item 15). & 9 \\
\hline Additional analysis & 23 & Give results of additional analyses, if done (e.g., sensitivity or subgroup analyses, meta-regression [see Item 16]). & 8 \\
\hline \multicolumn{4}{|l|}{ DISCUSSION } \\
\hline Summary of evidence & 24 & $\begin{array}{l}\text { Summarize the main findings including the strength of evidence for each main outcome; consider their relevance to } \\
\text { key groups (e.g., healthcare providers, users, and policy makers). }\end{array}$ & 9-10 \\
\hline Limitations & 25 & $\begin{array}{l}\text { Discuss limitations at study and outcome level (e.g., risk of bias), and at review-level (e.g., incomplete retrieval of } \\
\text { identified research, reporting bias). }\end{array}$ & 11 \\
\hline Conclusions & 26 & Provide a general interpretation of the results in the context of other evidence, and implications for future research. & $12-13$ \\
\hline \multicolumn{4}{|l|}{ FUNDING } \\
\hline Funding & 27 & $\begin{array}{l}\text { Describe sources of funding for the systematic review and other support (e.g., supply of data); role of funders for the } \\
\text { systematic review. }\end{array}$ & $\begin{array}{l}\text { N/A; not } \\
\text { funded }\end{array}$ \\
\hline
\end{tabular}

Fig. 2 PRISMA Checklist

samples (any CVD-related diagnoses) and homogeneous samples (e.g. coronary disease, heart failure, and hypertension). Although we did not exclude any specific type of diabetes, all studies within this review included patients diagnosed with type 2 diabetes. The mean age was $57.32(S D=7.40)$. All studies included an aerobic exercise program, meeting the 2008 and 2018 Physical Activity Guidelines of a minimum of $150 \mathrm{~min}$ of 
Table 1 Study Characteristics

\begin{tabular}{|c|c|c|c|c|c|c|}
\hline $\begin{array}{l}\text { First author, year } \\
\text { (superscript = references) }\end{array}$ & Sample size & Duration & Chronic disease & Type & Age in years (mean) & Location \\
\hline Gokal et al., 2016 [35] & 50 & 12 weeks & cancer & breast & 52.00 & home-based \\
\hline Huang et al., 2015 [36] & 159 & 12 weeks & cancer & breast & 48.27 & home-based \\
\hline Cadmus et al., 2009 [37] & 50 & 24 weeks & cancer & breast & 55.80 & home-based \\
\hline Courneya et al., 2009 [38] & 122 & 12 weeks & cancer & lymphoma & 53.20 & clinic-based \\
\hline Segal et al., 2008 [39] & 81 & 24 weeks & cancer & prostate & 65.75 & clinic-based \\
\hline Al-Majid et al., 2015 [40] & 14 & 12 weeks & cancer & breast & 50.30 & clinic-based \\
\hline Courneya et al., 2008 [41] & 242 & 17 weeks & cancer & breast & 50.00 & clinic-based \\
\hline Dodd et al., 2010 [42] & 119 & 12 weeks & cancer & breast, colorectal, or ovarian & 50.50 & home-based \\
\hline Duijts et al., 2012 [43] & 422 & 12 weeks & cancer & breast & 48.20 & home-based \\
\hline Giallauria et al., 2015 [44] & 94 & 52 weeks & cancer & breast & 53.50 & clinic-based \\
\hline Nikander et al., 2007 [45] & 28 & 12 weeks & cancer & breast & 53.00 & clinic-based \\
\hline Pickett et al., 2002 [46] & 52 & 12 weeks & cancer & breast & 52.00 & home-based \\
\hline Shang et al., 2012 [47] & 126 & 20 weeks & cancer & breast, colorectal, or prostate & 60.20 & clinic-based \\
\hline Courneya et al., 2003 [48] & 102 & 16 weeks & cancer & colorectal & 60.00 & home-based \\
\hline Lian et al., 2014 [49] & 330 & 12 weeks & CVD & coronary artery disease & 62.30 & home-based \\
\hline Li et al., 2015 [50] & 77 & 12 weeks & CVD & CVD & 80.68 & home-based \\
\hline Salvetti et al., 2008 [51] & 39 & 12 weeks & CVD & coronary disease & 53.00 & home-based \\
\hline Gary et al., 2011 [52] & 24 & 12 weeks & CVD & heart failure & 60.00 & home-based \\
\hline Guimaraes et al., 2014 [53] & 32 & 12 weeks & CVD & hyper-tension & 53.70 & clinic-based \\
\hline Houle et al., 2011 [54] & 65 & 52 weeks & CVD & acute coronary syndrome & 59.00 & home-based \\
\hline Kitzman et al., 2010 [55] & 53 & 16 weeks & CVD & heart failure & 70.00 & clinic-based \\
\hline Lee et al., 2015 [56] & 80 & 12 weeks & diabetes & type 2 & 56.08 & home-based \\
\hline Church et al., 2010 [57] & 113 & 36 weeks & diabetes & type 2 & 55.80 & clinic-based \\
\hline Dela et al., 2004 [58] & 24 & 12 weeks & diabetes & type 2 & 51.50 & home-based \\
\hline Balducci et al., 2014 [59] & 127 & 48 weeks & diabetes & type 2 & 60.00 & clinic-based \\
\hline Negri et al., 2010 [60] & 60 & 16 weeks & diabetes & type 2 & 65.70 & clinic-based \\
\hline Nicolucci et al., 2012 [61] & 606 & 49 weeks & diabetes & type 2 & 60.00 & clinic-based \\
\hline Sigal et al., 2007 [62] & 251 & 22 weeks & diabetes & type 2 & 53.50 & clinic-based \\
\hline Nam et al., 2012 [63] & 140 & 24 weeks & diabetes & type 2 & 56.39 & clinic-based \\
\hline Tessier et al., 2000 [64] & 39 & 16 weeks & diabetes & type 2 & 69.40 & clinic-based \\
\hline
\end{tabular}

moderate-intensity aerobic activity per week. Half of the studies included were clinic-based ( $n=16,53.3 \%)$. The pooled adherence rates for clinic-based and home-based programs were $74 \%$ [95\% CI, 0.65, 0.82] and $80 \%$ [95\% CI, 0.65, 0.91], respectively. Across conditions, there was greater variability for the number of clinic-based programs: cancer $(n=7,50.0 \%)$, CVD $(n=2,28.6 \%)$, and diabetes $(n=7,77.8 \%)$. The mean length of the intervention was 20 weeks (range 12 to 52 weeks). Of the 30 studies, 12 studies report the use of intention-to-treat (ITT) method for missing data, 13 studies report not using ITT, and 5 studies did not confirm the type of analysis used.

\section{Dropout and adherence rates of the interventions}

Overall, the un-weighted average adherence rate was $77 \%$ and the dropout rate was $7.0 \%$. No significant differences were found between the three chronic diseases. The pattern of data merely suggest that cancer patients had greater variability in adherence and dropout (adherence: $\min .=30.1 \%$, $\max .=92.9 \%$; dropout: $\min .=0 \%$, $\max .=40.1 \%$ ), compared to CVD (adherence: $\min .=$ $80.9 \%, \max .=100 \%$; dropout: $\min .=0 \%, \max .=28.1 \%$ ) and diabetes (adherence: $\min .=48.6 \%, \quad \max .=100 \%$; dropout: $\min .=0 \%, \max .=27.1 \%)$. We also did not see differences in adherence rates between strictly clinic-based (74\% [95\% CI, 0.65, 0.82]) and home-based 
(80\% [95\% CI, 0.65, 0.91]) aerobic exercise programs. Fig. 3 reports the meta-analysis estimates on the pooled difference in dropout rates between the intervention and control group for all studies and studies stratified by disease type (i.e., cancer, CVD, and diabetes). The estimated pooled difference in dropout rates based on all studies who reported dropout rates for both the intervention and control groups $(n=26)$ was 0.03 (95\% confidence interval $[\mathrm{CI}]=-0.03,0.08)$, denoting a lack of difference in dropout rates between intervention and control groups. Subgroup meta-analysis revealed no statistical significance as well. The estimated pooled differences in dropout rates based on studies with cancer $(n=12)$, CVD $(n=7)$, and type 2 diabetes $(\mathrm{n}=7)$ patients were $0.05(95 \% \mathrm{CI}$ $\left.[-0.04,0.14] ; \mathrm{I}^{2}=92.7 \%\right), 0.04$ (95\% CI $[-0.09,0.00]$; $\left.\mathrm{I}^{2}=0.0 \%\right)$, and $0.03\left(95 \%\right.$ CI $[-0.04,0.10], \mathrm{I}^{2}=$ $73.9 \%)$. The $\mathrm{I}^{2}$ did show substantial heterogeneity for dropout among the sub-diseases. Meta-regression revealed no difference in the differential dropout rates between intervention and control groups across studies stratified by disease type, intervention duration, ITT status, age, or intervention setting. The $p$-values for the Begg's test and Egger's test were 0.13 and 0.85 , respectively, denoting a lack of publication bias.
Figure 4 reports the meta-analysis estimates on the pooled adherence rate in the intervention group for all studies and studies stratified by disease type. The estimated pooled adherence rate based on all studies $(n=30)$ was $0.77(95 \% \mathrm{CI}=0.68,0.84)$ in the intervention group. The estimated pooled adherence rates for patients in the intervention groups of the cancer $(n=14)$, CVD $(n=7)$, and type 2 diabetes $(n=9)$ studies were $0.65\left(95 \%\right.$ CI $\left.[0.52,0.78] ; \mathrm{I}^{2}=94.30 \%\right), 0.90(95 \%$ CI $\left.[0.83,0.96] ; \mathrm{I}^{2}=60.02 \%\right)$, and $0.80(95 \% \mathrm{CI}[0.71,0.88]$; $\left.\mathrm{I}^{2}=85.86 \%\right)$, respectively. The $\mathrm{I}^{2}$ showed substantial heterogeneity among adherence rates for the three targeted diseases. Meta-regression found no difference in the pooled adherence rate in the intervention group across studies stratified by disease type, intervention duration, ITT status, age, or intervention setting. The $p$-values for the Begg's test and Egger's test were 0.62 and 0.13 , respectively, denoting a lack of publication bias.

\section{Discussion}

Individuals living with chronic disease must cope with a plethora of unique barriers to exercise (e.g., disease-specific symptoms, comorbidities, fatigue) that are irrelevant for healthy populations. The purpose of this systematic review

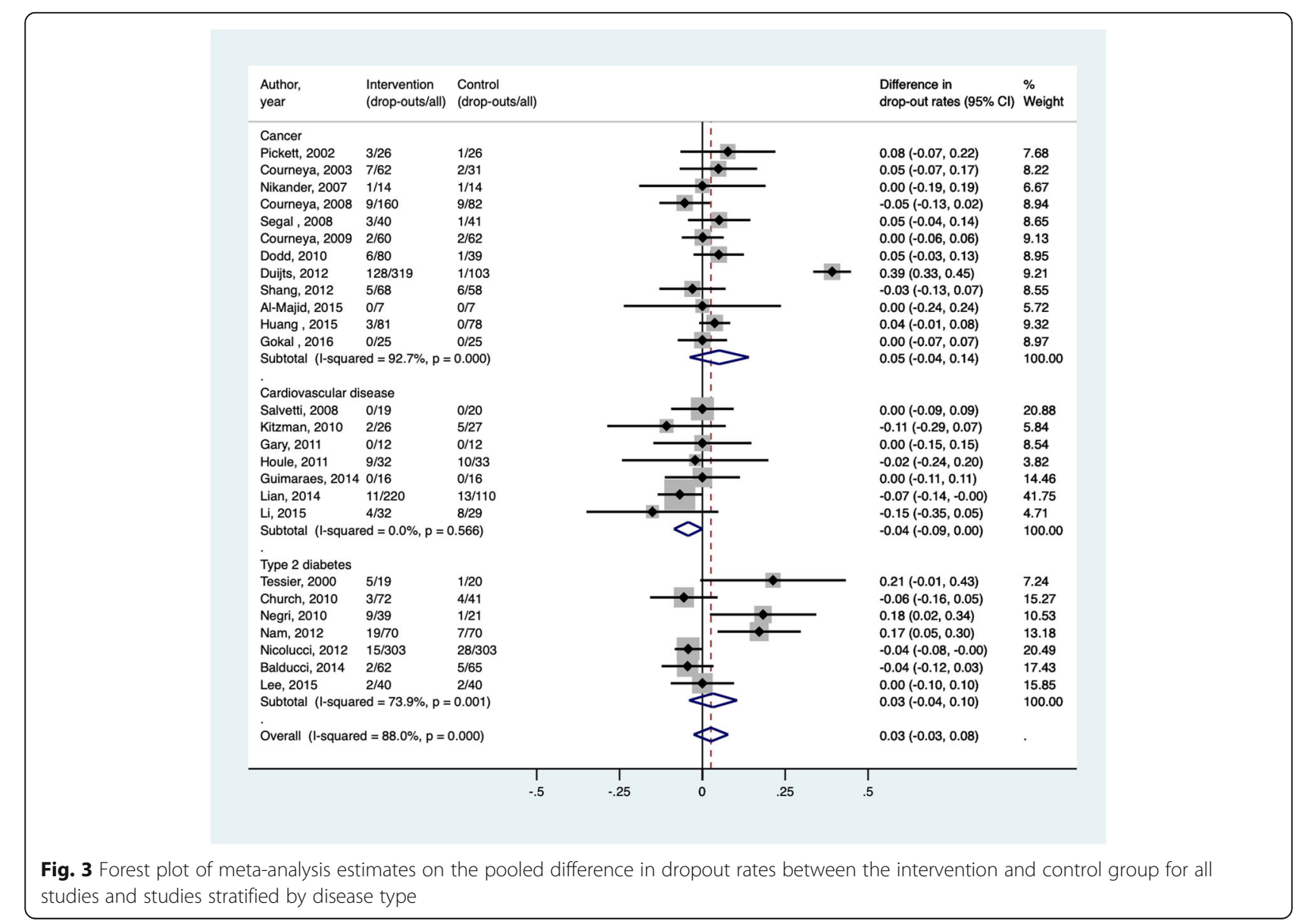




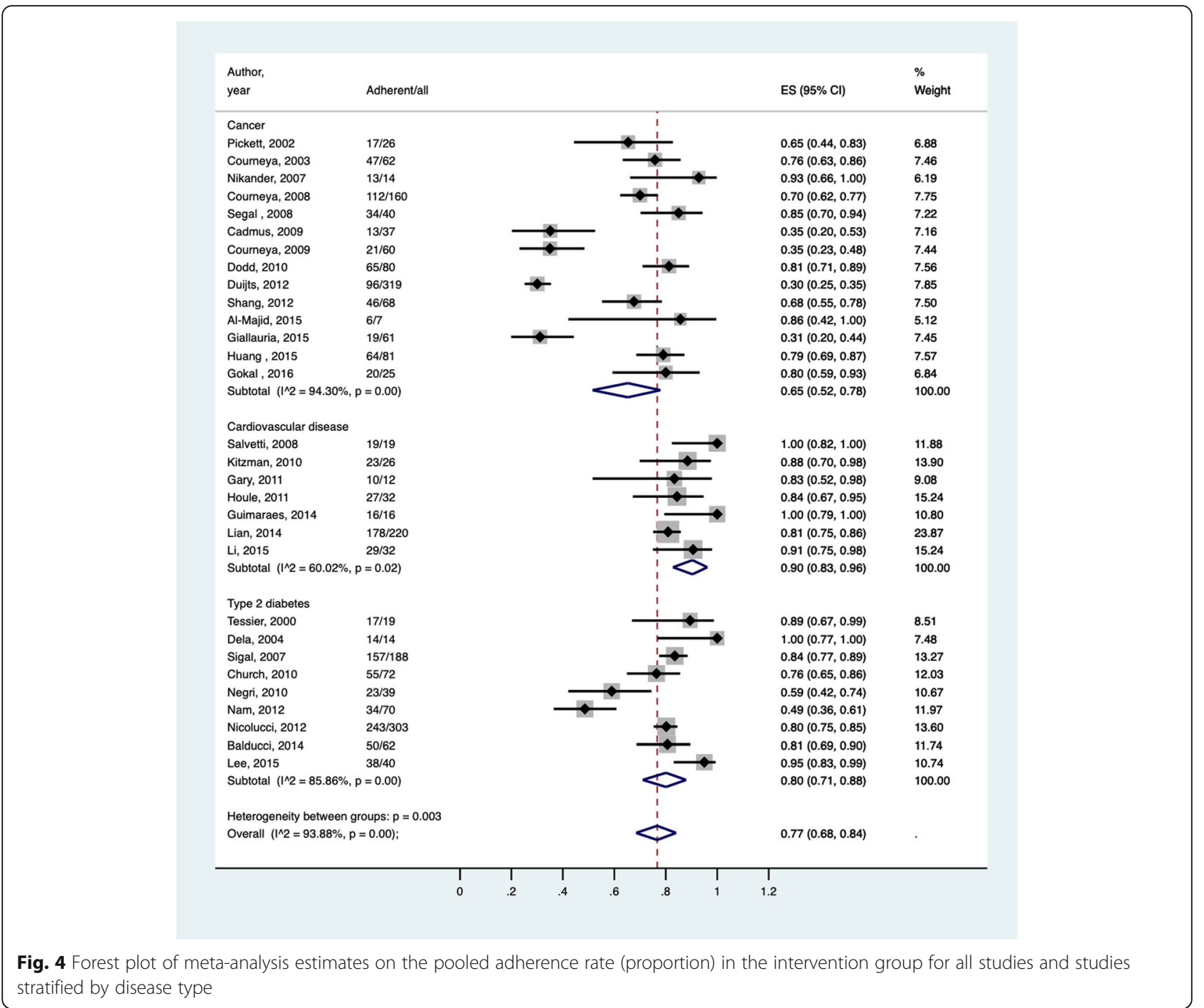

and meta-analysis was to examine the potential differences in adherence and dropout rates of aerobic physical activity interventions among people with chronic disease. We hypothesized that cancer would have the lowest adherence rates, based upon the low percentage of those with cancer who follow aerobic physical activity recommendations and given the known long-term effects of cancer treatment [21]. Contrary to our hypothesis, no statistically significant differences were found between the three chronic diseases. In addition, we did not see differences in adherence rates between strictly clinic-based (74\% [95\% CI, 0.65, 0.82]) and home-based (80\% [95\% CI, 0.65, 0.91]) aerobic exercise programs. These findings suggest that home-based programs may be just as feasible and perhaps, equally engaging, as programs designed with more professional supervision in rehabilitation clinics and research settings.

Overall, adherence to the exercise prescription (e.g. meeting aerobic physical activity guidelines) was $77 \%$. It is important to note that nearly half $(43.3 \%)$ of adherence data reported herein was not based on ITT (calculations only used data from those who completed studies). Failure to adopt ITT methods can inflate primary outcomes in RCTs [26], although ITT status did not appear to contribute to substantive differences in adherence across chronic conditions. The results suggest adherence to the physical activity guidelines is highly feasible among chronic disease populations, in both clinic and home-based physical activity interventions.

Given the substantial heterogeneity in adherence rates across studies within each targeted sub-population, it seems worthwhile to consider new approaches for increasing continued exercise engagement among individuals who may have particular difficulty (falling well short of the average). For example, a precision behavioral medicine approach could begin to identify "red flags," (scores indicating below-average functioning) to facilitate 
decisions about the appropriate type or timing of interventions. Such an approach can be used to inform supplemental strategies focusing on the interaction among disease symptomology with physical functioning (e.g., mobility limitations) and psychological functioning (e.g., self-efficacy beliefs), all of which can change, and contributes to variability in adherence. In support of this, Pedersen and Saltin (2006) found that in general, adherence to physical activity prescriptions among patients with chronic disease are more likely to occur when they are individualized to the patient, initially supervised, and include both aerobic and strength components [27]. Interestingly, Wong, McAuley, and Trinh (2018) reviewed physical activity program preferences among cancer survivors and found a wide variation of preferences, suggesting that tailored programs may optimize program adherence [28].

Although the studies herein provide greater insight into aerobic physical activity adherence and dropout rates among chronic disease populations, insufficient information is available to explain our null findings. Many studies have targeted exercise determinants, yet evidence has not pointed to a consistent set of factors associated with adherence to physical activity guidelines or exercise programs. For example, using a broad framework incorporating many social and ecological factors, Kampshoff et al. [29] found that only one's past exercise experience was associated with adherence among cancer survivors. Few systematic reviews exist, focused on determinants of exercise adherence for people with CVD and diabetes. Daly et al. [30] identified demographic factors as well as perceived benefits and leisure-time physical activity to be associated with non-adherence among cardiac rehabilitation patients. However, the authors pointed out that there were many methodological limitations of the studies in their sample (particularly a lack of randomized controlled designs), making it unclear as to which factors are associated with adherence levels among people with CVD. Allen [31] conducted a review examining exercise adherence in populations with diabetes and found self-efficacy measures to be predictive of exercise initiation and maintenance. However, with most studies included in the review, exercise was not the primary outcome. Rather the primary interventions were self-care regimens that included an exercise monitoring component. The limited research in this area and the variability among studies that do exist make it difficult to assess the most robust determinants of physical activity adherence among chronic disease populations.

Although it is unclear which factors reliably contribute to exercise adherence, specifically among people living with chronic disease, some factors seem to be unrelated (exercise modality, location). For example, Yang et al. [32] compared the effectiveness of aerobic and resistance exercise in populations with diabetes and found no evidence that either modality resulted in more favorable health outcomes. Yang et al. concluded that instead of focusing on the most preferred type of exercise, there should be a greater emphasis on getting chronic disease populations to remain physically active. In two other reviews of cardiac rehabilitation patients' program adherence, Anderson et al. [33] and Dalal et al. [34] found that home- and center-based programs were equally effective in improving health-related quality of life. Anderson et al. also found programs that included self-regulatory factors (i.e. self-monitoring, action planning) resulted in the greatest levels of program adherence. Together, our findings coupled with prior research underscore the problem of exercise adherence. Perhaps a more patient-centered perspective and targeting self-regulatory deficiencies and strengths may benefit people with chronic conditions more so than generic exercise interventions designed to overcome common barriers.

There are several limitations that should be considered when interpreting the results of this study and should be addressed in future research. First, the exercise prescriptions varied in duration, intensity and complexity. In addition, this study only targeted trials lasting at least three months (again, for consistency purposes, to align with established data regarding exercise program dropout). This resulted in only 30 studies being used for the review and limited our power to test potential moderating characteristics of sample attributes and trial design.

\section{Conclusion}

The findings of this review suggest that among people with different chronic conditions participating in aerobic physical activity interventions, there is consistency in the extent to which they are non-adherent. The overall rate of adherence was $77 \%$ to the physical activity programs, and the dropout rate was $7 \%$, suggesting that people with chronic conditions are capable of sustaining physical activity for $3+$ months, under different degrees of supervision, at levels sufficient for health benefits. Future researchers and healthcare providers should continue to develop adherence-promotion strategies that account for the shared barriers across chronic disease populations, as well as the known variability within these sub-populations.

\footnotetext{
Abbreviations

Cl: confidence interval; CINAHL: Cumulative Index to Nursing and Allied Health Literature; CVD: cardiovascular disease; ITT: intention-to-treat; MET: metabolic equivalent task; PRISMA: preferred reporting items for systematic review and meta-analysis; PRISMA-P: preferred reporting items for systematic review and meta-analysis protocols; RCT: randomized controlled trial
} 


\section{Acknowledgements}

Not applicable.

\section{Funding}

Not applicable.

\section{Availability of data and materials}

The datasets used and/or analysed during the current study are available from the corresponding author on reasonable request.

\section{Authors' contributions}

TB carried out the data collection, study design, data analysis and interpretation, and manuscript drafting. MJ carried out the data analysis and interpretation. RA carried out the data analysis and interpretation. LT carried out the data interpretation and manuscript reviewing. MM carried out the data interpretations and manuscript reviewing. SPM carried out the study conceptualization, study design, data interpretation, and manuscript drafting. All authors read and approved the final manuscript.

\section{Ethics approval and consent to participate}

Not applicable.

\section{Consent for publication}

Not applicable.

\section{Competing interests}

The authors declare that they have no competing interests.

\section{Publisher's Note}

Springer Nature remains neutral with regard to jurisdictional claims in published maps and institutional affiliations.

\section{Author details}

${ }^{1}$ University of Illinois at Urbana-Champaign, Champaign, USA. ${ }^{2}$ University of Toronto, Toronto, Canada. ${ }^{3}$ University of Delaware, Newark, Delaware, USA.

Received: 18 January 2019 Accepted: 22 April 2019

Published online: 24 May 2019

\section{References}

1. Ward BW, Schiller JS, Goodman RA. Peer reviewed: multiple chronic conditions among U.S. adults: a 2012 update. Prev Chronic Dis. 2014;11.

2. Stevinson C, Lawlor DA, Fox KR. Exercise interventions for cancer patients: systematic review of controlled trials. Cancer Causes Control. 2004;15(10): 1035-56.

3. Taylor RS, Brown A, Ebrahim S, et al. Exercise-based rehabilitation for patients with coronary heart disease: systematic review and meta-analysis of randomized controlled trials. Am J Med. 2004;116(10):682-92.

4. Zanuso S, Jimenez A, Pugliese G, Corigliano G, Balducci S. Exercise for the management of type 2 diabetes: a review of the evidence. Acta Diabetol. 2010;47(1):15-22.

5. Centers for Disease Control and Prevention (CDC). Physical activity and health. https://www.cdc.gov/physicalactivity/basics/pa-health/index. htm\#ReducedCancer. Accessed December 14, 2018.

6. Bize R, Johnson JA, Plotnikoff RC. Physical activity level and health-related quality of life in the general adult population: a systematic review. Prev Med. 2007:45(6):401-15.

7. Haskell WL, Lee IM, Pate RR, et al. Physical activity and public health: updated recommendation for adults from the American College of Sports Medicine and the American Heart Association. Circulation. 2007;116(9):1081-93.

8. Hair B, Hayes S, Tse C, Bell M, Olshan A. Racial differences in physical activity among breast cancer surviviors: implications for breast cancer care. Cancer. 2014;120(14):2174-82.

9. Valero-Elizondo J, Salami JA, Osondu CU, et al. Economic impact of moderate-vigorous physical activity among those with and without established cardiovascular disease: 2012 medical expenditure panel survey. J Am Heart Assoc. 2016;5(9):e003614.

10. Janevic MR, McLaughlin SJ, Connell CM. Overestimation of physical activity among a nationally representative sample of underactive individuals with diabetes. Med Care. 2012;50(5):441-5.
11. Go AS, Mozaffarian D, Roger VL, et al. Heart disease and stroke statistics - 2014 update: a report from the American Heart Association. Circulation. 2014;129(3):e28.

12. Eijsvogels TM, Thompson PD. Exercise is medicine: at any dose? Journal of American Medical Association. 2015;314(18):1915-6.

13. Naci $\mathrm{H}$, loannidis JP. Comparative effectiveness of exercise and drug interventions on mortality outcomes: metaepidemiological study. The British Medical Journal. 2013;347:f5577.

14. Conn VS, Valentine JC, Cooper HM. Interventions to increase physical activity among aging adults: a meta-analysis. Ann Behav Med. 2002;24(3): 190-200.

15. Gerritsen JK, Vincent AJ. Exercise improves quality of life in patients with cancer: a systematic review and meta-analysis of randomised controlled trials. Br J Sports Med. 2015:796-803.

16. Holmes MD, Chen WY, Feskanich D, Kroenke CH, Colditz GA. Physical activity and survival after breast cancer diagnosis. Journal of American Medical Association. 2005;293(20):2479-86.

17. Anderson L, Oldridge N, Thompson DR, Zwisler AD, Rees K, Martin N, Taylor RS. Exercise-based cardiac rehabilitation for coronary heart disease: Cochrane systematic review and meta-analysis. J Am Coll Cardiol. 2016; 67(1):1-12.

18. Umpierre D, Ribeiro PA, Kramer CK, et al. Physical activity advice only or structured exercise training and association with $\mathrm{HbA} 1 \mathrm{c}$ levels in type 2 diabetes: a systematic review and meta-analysis. Journal of American Medical Association. 2011;305(17):1790-9.

19. Hu G, Jousilahti P, Barengo NC, Qiao Q, Lakka TA, Tuomilehto J. Physical activity, cardiovascular risk factors, and mortality among Finnish adults with diabetes. Diabetes Care. 2005;28(4):799-805.

20. Pedersen BK, Saltin B. Exercise as medicine-evidence for prescribing exercise as therapy in 26 different chronic diseases. Scand J Med Sci Sports. 2015;25(S3):1-72.

21. Hofman M, Ryan JL, Figueroa-Moseley CD, Jean-Pierre P, Morrow GR Cancer-related fatigue: the scale of the problem. Oncologist. 2007;12(1): 4-10.

22. Dishman RK. Increasing and maintaining exercise and physical activity Behav Ther. 1991:22(3):345-78.

23. Mullen SP. Perceptions of change and certainty regarding the self-asexerciser: a multistudy report. Journal of Sport and Exercise Psychology. 2011;33(5):710-33.

24. von Gruenigen VE, Courneya KS, Gibbons HE, Kavanagh MB, Waggoner SE, Lerner E. Feasibility and effectiveness of a lifestyle intervention program in obese endometrial cancer patients: a randomized trial. Gynecol Oncol. 2008; 109(1):19-26.

25. Moher D, Liberati A, Tetzlaff J, Altman DG. The PRISMA group. Preferred reporting items for systematic reviews and meta-analyses: the PRISMA statement. Int J Surg. 2010;8(5):336-41.

26. Kraemer HC, Wilson GT, Fairburn CG, Agras WS. Mediators and moderators of treatment effects in randomized clinical trials. Arch Gen Psychiatry. 2002; 59(10):877-83.

27. Pedersen BK, Saltin B. Evidence for prescribing exercise as therapy in chronic disease. Scand J Med Sci Sports. 2006;16(S1):3-63.

28. Wong JN, McAuley E, Trinh L. Physical activity programming and counseling preferences among cancer survivors: a systematic review. Int J Behav Nutr Phys Act. 2018;15(1):48.

29. Kampshoff CS, Jansen F, van Mechelen W, May AM, Brug J, Chinapaw MJ, Buffart LM. Determinants of exercise adherence and maintenance among cancer survivors: a systematic review. Int J Behav Nutr Phys Act. 2014;11(1):80

30. Daly J, Sindone AP, Thompson DR, Hancock K, Chang E, Davidson P. Barriers to participation in and adherence to cardiac rehabilitation programs: a critical literature review. Prog Cardiovasc Nurs. 2002;17(1):8-17.

31. Allen NA. Social cognitive theory in diabetes exercise research: an integrative literature review. The Diabetes Educator. 2004;30(5):805-19.

32. Yang Z, Scott CA, Mao C, Tang J, Farmer AJ. Resistance exercise versus aerobic exercise for type 2 diabetes: a systematic review and meta-analysis. Sports Med. 2014:44(4):487-99.

33. Anderson L, Taylor RS. Cardiac rehabilitation for people with heart disease: an overview of Cochrane systematic reviews. Int J Cardiol. 2014;177(2):348-61.

34. Dalal HM, Zawada A, Jolly K, Moxham T, Taylor RS. Home based versus Centre based cardiac rehabilitation: Cochrane systematic review and metaanalysis. Br Med J. 2010;340:b5631. 
35. Gokal K, Wallis D, Ahmed S, Boiangiu I, Kancherla K, Munir F. Effects of a self-managed home-based walking intervention on psychosocial health outcomes for breast cancer patients receiving chemotherapy: a randomised controlled trial. Support Care Cancer. 2016;24(3):1139-66.

36. Huang HP, Wen FH, Tsai JC, et al. Adherence to prescribed exercise time and intensity declines as the exercise program proceeds: findings from women under treatment for breast cancer. Support Care Cancer. 2015;23(7): 2061-71.

37. Cadmus LA, Salovey P, Yu H, Chung G, Kasl S, Irwin ML. Exercise and quality of life during and after treatment for breast cancer: results of two randomized controlled trials. Psycho-Oncology: Journal of the Psychological, Social and Behavioral Dimensions of Cancer. 2009;18(4):343-52.

38. Courneya KS, Sellar CM, Stevinson C, et al. Randomized controlled trial of the effects of aerobic exercise on physical functioning and quality of life in lymphoma patients. Proc Am Soc Clin Oncol. 2009.

39. Segal RJ, Reid RD, Courneya KS, et al. Randomized controlled trial of resistance or aerobic exercise in men receiving radiation therapy for prostate cancer. J Clin Oncol. 2009;27(3):344-51.

40. Al-Majid S, Wilson LD, Rakovski C, Coburn JW. Effects of exercise on biobehavioral outcomes of fatigue during cancer treatment: results of a feasibility study. Biological Research for Nursing. 2015;17(1):40-8.

41. Courneya KS, McKenzie DC, Mackey JR, et al. Moderators of the effects of exercise training in breast cancer patients receiving chemotherapy. Cancer. 2008;112(8):1845-53

42. Dodd MJ, Cho MH, Miaskowski C, et al. A randomized controlled trial of home-based exercise for cancer-related fatigue in women during and after chemotherapy with or without radiation therapy. Cancer Nurs. 2010;33(4): 245-57.

43. Duijts SF, van Beurden M, Oldenburg HS, et al. Efficacy of cognitive behavioral therapy and physical exercise in alleviating treatment-induced menopausal symptoms in patients with breast cancer: results of a randomized, controlled, multicenter trial. J Clin Oncol. 2012;30(33):4124-33.

44. Giallauria $F$, Gentile $M$, Chiodini $P$, et al. Exercise training reduces high mobility group box-1 protein levels in women with breast cancer: findings from the DIANA-5 study. Monaldi Arch Chest Dis. 2015;82(2):61-7.

45. Nikander R, Sievänen $\mathrm{H}$, Ojala $\mathrm{K}$, et al. Effect of a vigorous aerobic regimen on physical performance in breast cancer patients - a randomized controlled pilot trial. Acta Oncol. 2007:46(2):181-6.

46. Pickett M, Mock V, Ropka ME, Cameron L, Coleman M, Podewils L. Adherence to moderate-intensity exercise during breast cancer therapy. Cancer Pract. 2002;10(6):284-92.

47. Shang J, Wenzel J, Krumm S, Griffith K, Stewart K. Who will drop out \& who will drop in, exercise adherence in a RCT among patients receiving active cancer treatment. Cancer Nurs. 2012;35(4):312-22.

48. Courneya K, Friedenreich C, Quinney H, Fields A, Jones L, Fairey A. A randomized trial of exercise and quality of life in colorectal cancer survivors. European Journal of Cancer Care. 2003;12(4):347-57.

49. Lian $X Q$, Zhao $D$, Zhu $M$, et al. The influence of regular walking at different times of day on blood lipids and inflammatory markers in sedentary patients with coronary artery disease. Prev Med. 2014;58:64-9.

50. Li X, Xu S, Zhou L, Li R, Wang J. Home-based exercise in older adults recently discharged from the hospital for cardiovascular disease in China: randomized clinical trial. Nurs Res. 2015;64(4):246-55.

51. Salvetti XM, Oliveira JA, Servantes DM, Vincenzo de Paola AA. How much do the benefits cost? Effects of a home-based training programme on cardiovascular fitness, quality of life, programme cost and adherence for patients with coronary disease. Clin Rehabil. 2008;22(10-11):987-96.

52. Gary RA, Cress ME, Higgins MK, Smith AL, Dunbar SB. Combined aerobic and resistance exercise program improves task performance in patients with heart failure. Arch Phys Med Rehabil. 2011;92(9):1371-81.

53. Guimaraes GV, de Barros Cruz LG, Fernandes-Silva MM, Dorea EL, Bocchi EA. Heated water-based exercise training reduces 24-hour ambulatory blood pressure levels in resistant hypertensive patients: a randomized controlled trial (HEx trial). Int J Cardiol. 2014;172(2):434-41.

54. Houle J, Doyon O, Vadeboncoeur N, Turbide G, Diaz A, Poirier P. Innovative program to increase physical activity following an acute coronary syndrome: randomized controlled trial. Patient Educ Couns. 2011;85(3):e237-44.

55. Kitzman DW, Brubaker PH, Morgan TM, Stewart KP, Little WC. Exercise training in older patients with heart failure and preserved ejection fraction: a randomized, controlled, single-blind trial. Circ Heart Fail. 2010;3(6):659-67.
56. Lee SF, Pei D, Chi MJ, Jeng C. An investigation and comparison of the effectiveness of different exercise programmes in improving glucose metabolism and pancreatic $\beta$ cell function of type 2 diabetes patients. Int J Clin Pract. 2015;69(10):1159-70.

57. Church TS, Blair SN, Cocreham S, et al. Effects of aerobic and resistance training on hemoglobin A1c levels in patients with type 2 diabetes: a randomized controlled trial. J Am Med Assoc. 2010;304(20):2253-62.

58. Dela F, von Linstow ME, Mikines KJ, Galbo H. Physical training may enhance $\beta$-cell function in type 2 diabetes. American Journal of PhysiologyEndocrinology and Metabolism. 2004;287(5):E1024-31.

59. Balducci S, Vulpiani MC, Pugliese L, et al. Effect of supervised exercise training on musculoskeletal symptoms and function in patients with type 2 diabetes: the italian diabetes exercise study. Acta Diabetol. 2014;51(4):647-54

60. Negri C, Bacchi E, Morgante $\mathrm{S}$, et al. Supervised walking groups to increase physical activity in type 2 diabetic patients. Diabetes Care. 2010;33(11):2333-5.

61. Nicolucci A, Balducci S, Cardelli P, et al. Relationship of exercise volume to improvements of quality of life with supervised exercise training in patients with type 2 diabetes in a randomised controlled trial: the italian diabetes and exercise study (IDES). Diabetologia. 2012;55(3):579-88.

62. Sigal RJ, Kenny GP, Boulé NG, et al. Effects of aerobic training, resistance training, or both on glycemic control in type 2 diabetes: a randomized trial. Ann Intern Med. 2007;147(6):357-69.

63. Nam S, Dobrosielski DA, Stewart KJ. Predictors of exercise intervention dropout in sedentary individuals with type 2 diabetes. Journal of Cardiopulmonary Rehabilitation and Prevention. 2012;32(6):370-8.

64. Tessier D, Ménard J, Fülöp T, et al. Effects of aerobic physical exercise in the elderly with type 2 diabetes mellitus. Arch Gerontol Geriatr. 2000;31(2):121-32.

\section{Ready to submit your research? Choose BMC and benefit from:}

- fast, convenient online submission

- thorough peer review by experienced researchers in your field

- rapid publication on acceptance

- support for research data, including large and complex data types

- gold Open Access which fosters wider collaboration and increased citations

- maximum visibility for your research: over $100 \mathrm{M}$ website views per year

At BMC, research is always in progress.

Learn more biomedcentral.com/submissions 